\title{
Editorial des Geschäftsführenden Herausgebers
}

Dieses Heft der Zeitschrift GRUPPENDYNAMIK und ORGANISATIONSBERATUNG ist das erste, das wir als Kölner Team herausgeben. Dabei haben wir zunächst dem bisherigen Geschäftsführenden Herausgeber Prof. Dr. Helmut E. Lück sowie Dr. Horst Heidbrink und Frau Heide Gebauer aus der Hagener Redaktion herzlich zu danken, die uns die Zeitschrift in vorzüglicher Ordnung übergeben haben. In der Zeit der Übergabe haben sie uns mit Rat und Tat und ihrer langjährigen Erfahrung unterstützend zur Seite gestanden.

Ich will an dieser Stelle kurz daran erinnern, was Gruppendynamik, so der frühere Titel der Zeitschrift, alles ist:

- Gruppendynamik ist empirische und experimentelle Kleingruppenforschung,

- Gruppendynamik ist das Feld, in dem die Aktionsforschung entstand,

- Gruppendynamik ist alles, was in und zwischen Gruppen geschieht,

- Gruppendynamik ist ein Spektrum von Interventionsmethoden mit den Zielen Selbsterfahrung, Beziehungsklärung und Aneignung von Interaktionskompetenzen,

- Gruppendynamik ist ein Teilaspekt aller Prozesse psychosozialer Beeinflussung wie Beratung, Psychotherapie, Selbstorganisation und Selbsthilfe, Supervision und Coaching, Mediation und Moderation, Training und Schulung, Unterricht und Anleitung, Erwachsenenbildung und Sozialisation generell,

- Gruppendynamik ist sichtbar in den situativen und gesellschaftlichen Kontexten, in denen diese Beeinflussungen stattfinden,

- Gruppendynamik ist Basis aller privaten und beruflichen Interaktionen.

Neben dem Themenkomplex Gruppendynamik soll in dieser Zeitschrift besonders der neue Schwerpunkt Organisationsberatung deutlich werden. Wir als neues Geschäftsführendes Herausgeber-Team nehmen uns vor, in diesen Feldern weiterhin Trends, Positionen, Theorien, Erkenntnisse und Konzepte aufzuzeigen und ihre Bedeutung für den Stand der wissenschaftlichen Diskussion wie auch für das praktische Handeln und Intervenieren sichtbar zu machen.

In diesem Anspruch bitten wir die Leserinnen und Leser dieser Zeitschrift weiterhin um ihre kritische Unterstützung.

Stephanie Haustein Susanne Nußbeck_Jörg Fengler 